\title{
Large-Eddy Simulation of Autoignition-Dominated Supersonic Combustion
}

\author{
Graham V. Candler* \\ Aerospace Engineering and Mechanics, University of Minnesota, Minneapolis, MN \\ Niccolo Cymbalist $†$ and Paul E. Dimotakis ${ }^{\ddagger}$ \\ California Institute of Technology, Pasadena, CA
}

\begin{abstract}
The simulation of low-speed combustion flows is well established. However, at highspeed conditions where radical formation and ignition delay are important, there is much less experience with turbulent combustion modeling. In the present work, a novel evolution variable manifold (EVM) approach of Cymbalist and Dimotakis ${ }^{1,2}$ is implemented in a production CFD code and preliminary RANS and large-eddy simulations are computed for a hydrogen combustion test case. The EVM approach solves a scalar conservation equation for the induction time to represent ignition delay. The state of the combustion products is tabulated as a function of density, energy, mixture fraction, and the evolution variable. A thermodynamically-consistent numerical flux function is developed and the approach for coupling the EVM table to CFD is discussed. Initial simulations show that the EVM approach produces good agreement with full chemical kinetics model simulations. Work remains to be done to improve the numerical stability, extend the grid, and increase the order of accuracy of the simulations.
\end{abstract}

\section{Introduction}

The accurate simulation of turbulent combustion in high-speed air-breathing engines is important for their design and optimization. The most promising approach for representing these complex flows is largeeddy simulations (LES), in which large-scale turbulent motion is resolved and small-scale effects are modeled. There are several widely-used approaches for the LES of combustion flows, but most were originally developed and applied to the study of low-speed combustion flows. In high-speed flows, a critical effect is ignition delay, in which a flowing fuel element is gradually decomposed until a critical level of radicals is produced, resulting in ignition, rapid oxidation, and heat release. Because of the high-speed conditions, this induction or ignition delay may take place over a very large physical distance in the combustor, and can dominate the overall engine performance.

Cymbalist and Dimotakis ${ }^{1,2}$ have shown that detailed chemical kinetics models for ethylene exhibit extremely large variation in the ignition delay time at relevant conditions in air. They propose a data-driven model in which the ignition delay and subsequent ignition and heat-release processes are represented with an induction-evolution variable. Experimental shock-tube data are used to build a response surface for this variable across the full range of possible conditions within a high-speed combustor. The solution of this variable, along with a variable that tracks the fuel-air mixture fraction, uniquely determines the thermochemical state of the combustion process. Such an approach promises to greatly reduce the cost and improve the accuracy of large-eddy simulations of reacting hydrocarbon flows. For cases in which a detailed chemical kinetics model is valid, it can be used to construct the response surface, rather than relying on experimental data.

The proposed induction-evolution manifold (EVM) approach has similarities to the flamelet-progress variable approach of Pierce and Moin, ${ }^{3}$ its extension to compressible flows, ${ }^{4}$ and others, but is developed

\footnotetext{
*Russell J. Penrose and McKnight Presidential Professor, Fellow AIAA

${ }^{\dagger}$ Graduate Research Assistant, Student Member AIAA

${ }^{\ddagger}$ Professor of Aeronautics \& Applied Physics, Fellow AIAA
} 
with a completely different set of assumptions and modeling limitations. Here, the representation of the induction, ignition, and heat release processes are valid across a complete range of relevant pressures and temperatures. Furthermore, the ignition delay process is calibrated so that it fits the available experimental data or detailed chemical kinetics model at all relevant conditions.

In the present paper, we modify an existing CFD $\operatorname{code}^{5}$ for the LES of compressible reacting flows to represent the thermo-chemical state of the fuel-air mixture with the induction-evolution variable and a mixture fraction variable. The focus is on the development of a numerical method that is rigorously consistent with the thermo-chemical model, so that the numerical flux function correctly reflects the variations in the thermodynamic state. We apply the proposed numerical flux function to the reacting hydrogen injection experiments of Gamba et al. ${ }^{6,7}$ using the hydrogen-air chemical kinetics model of Burke et al. ${ }^{8}$

\section{Induction-Evolution Variable Approach}

In this section, the induction-evolution variable manifold (EVM) approach is discussed briefly; additional details may be found in Cymbalist and Dimotakis. ${ }^{1,2}$ The EVM approach represents the decomposition of the fuel prior to ignition using an evolution variable, $\tau$. When $\tau$ reaches a critical value, the gas mixture has ignited and oxidation occurs. The power of this method is several fold. For hydrocarbons, detailed chemical kinetics models require a very large number of chemical species and a correspondingly daunting number of reactions. Yet, even the most sophisticated models for a simple hydrocarbon like ethylene $\left(\mathrm{C}_{2} \mathrm{H}_{4}\right)$ have a large uncertainty in the ignition delay time. Most of these species are radicals and are present at very small levels, yet they largely determine the induction time and the onset of oxidation. The EVM approach addresses this problem on two fronts: first, it uses an experimental database, or detailed kinetics model if accurate, to pre-compute the rate of radical formation and progress toward ignition; and secondly, it produces a tabulated database that can be used to determine the thermo-chemical state of the gas mixture at any point in the combustion process.

Critical to the EVM approach is the induction-evolution variable, $\tau$. This measures how far a fluid element has progressed toward ignition; $\tau$ varies depending on the local thermodynamic state, how far the induction-ignition process has progressed, and the rate of entrainment and mixing of the fuel. Within a fluid element in the combustion flow field, this variable can be represented as the integral of the rate of progress toward ignition

$$
\int_{0}^{t} \zeta\left(t_{\text {d.c. }}\right) d t=\tau, \quad 0<\tau<\kappa
$$

where $\zeta$ is the rate of change of the evolution variable, which is a function of $t_{\text {d.c. }}$, the instantaneous ignition delay time, and $\kappa$ is the value of $\tau$ at ignition. This approach extends the approach of Williams, ${ }^{9}$ but uses an exponential variation of the rate, $\zeta$, to capture the exponential growth of the radical pool prior to ignition..$^{1,2}$

The thermo-chemical state of the gas is determined by how far it has progressed toward ignition, and then once ignition has occurred, the rate at which heat is released as a function of the local state. With this approach, it is possible to tabulate the gas state as a function of the pressure, enthalpy, fuel mixture fraction, and induction-evolution variable. For purposes of CFD implementation, the gas state may also be tabulated in terms of density and energy rather than pressure and enthalpy. Thus, any thermodynamic variable, $\phi$, can be written as

$$
\phi=\phi(\rho, e, Z, \tau)
$$

For cases in which the entrainment rate of fuel into oxidizer, $\chi$, is important, the induction-evolution variable, $\tau$, also depends on $\chi$.

The EVM manifold is constructed in two steps. Post-ignition, it is populated using homogeneous, constant energy and density reactors, where the species concentration evolution is given by

$$
\frac{d y_{s}}{d t}=\dot{\omega}_{s}
$$

The reactors are initialized across the expected range of $\rho, e$ in the flow and are run for a number of flow times. The species mass fractions, $y_{s}$, overall reaction evolution rate of the combustion products

$$
\zeta_{c}=\sum_{j} \frac{\dot{\omega}_{p, j}}{\left(y_{p, j}\right)_{\mathrm{eq}}}
$$


and partial derivatives

$$
\frac{\partial \zeta_{c}}{\partial e}, \quad \frac{\partial \zeta_{c}}{\partial \tau_{c}}, \quad \frac{\partial \zeta_{c}}{\partial \rho}
$$

are extracted and stored in the table as a function of $\rho, e, Z$ and $\tau=\tau_{c}$,

$$
\tau_{c}=\sum_{j} \frac{y_{p, j}(t)}{\left(y_{p, j}\right)_{\mathrm{eq}}}
$$

If a reactor does not ignite, ignition is forced by introducing a radical pool using

$$
\frac{d y_{s}}{d t}=\dot{\omega}_{s}, \quad y_{s}(t=0)=y_{s, i}
$$

where $\left(y_{s, i}\right)$ is the composition just prior to ignition of a reactor that is mixture fraction matched, but at conditions that lead to autoignition. $y_{s, i}$ is pre-computed and tabulated as a function of mixture fraction.

Prior to ignition, the reaction evolution rate $\zeta$ is given by the expression

$$
\zeta_{i}\left(\tau_{i}, t_{\mathrm{d}, \mathrm{c}}, \kappa, \tau_{i, 0}\right)=\frac{\tau_{i}}{t_{\mathrm{d}, \mathrm{c}}} \log \left(\frac{\kappa}{\tau_{i, 0}}\right)
$$

where $\tau_{i, 0}$ is an assumed background value of the induction evolution-variable, $\kappa / \tau_{i, 0}$ is the dynamic range of the radical-pool concentration during induction, and $t_{\mathrm{d}, \mathrm{c}}$ is the characteristic ignition delay.

For example, Figure 1 plots the rate of change of the evolution variable, $\zeta$, and the water mass fraction as a function of $\rho, Z, \tau$ at a fixed value of energy for the EVM table constructed to represent the hydrogen-air experiments of Gamba et al. ${ }^{6,7}$ The detailed chemical kinetics model of Burke et al. ${ }^{8}$ was used to construct the table.

Complete details of the EVM approach are given in Refs. 1 and 2. For the present purpose of developing a self-consistent numerical method to solve the EVM equations, the key idea is that for a given $\rho, e, Z, \tau$ (and possibly $\chi$ ), it is possible to uniquely determine the thermodynamic state and chemical composition of the gas. For a given combination of fuel and oxidizer streams and a range of flow field conditions, this function can be tabulated for use by a computational fluid dynamics code. The remainder of the paper considers how to formulate a numerical flux function for this tabular representation of the thermodynamic state.
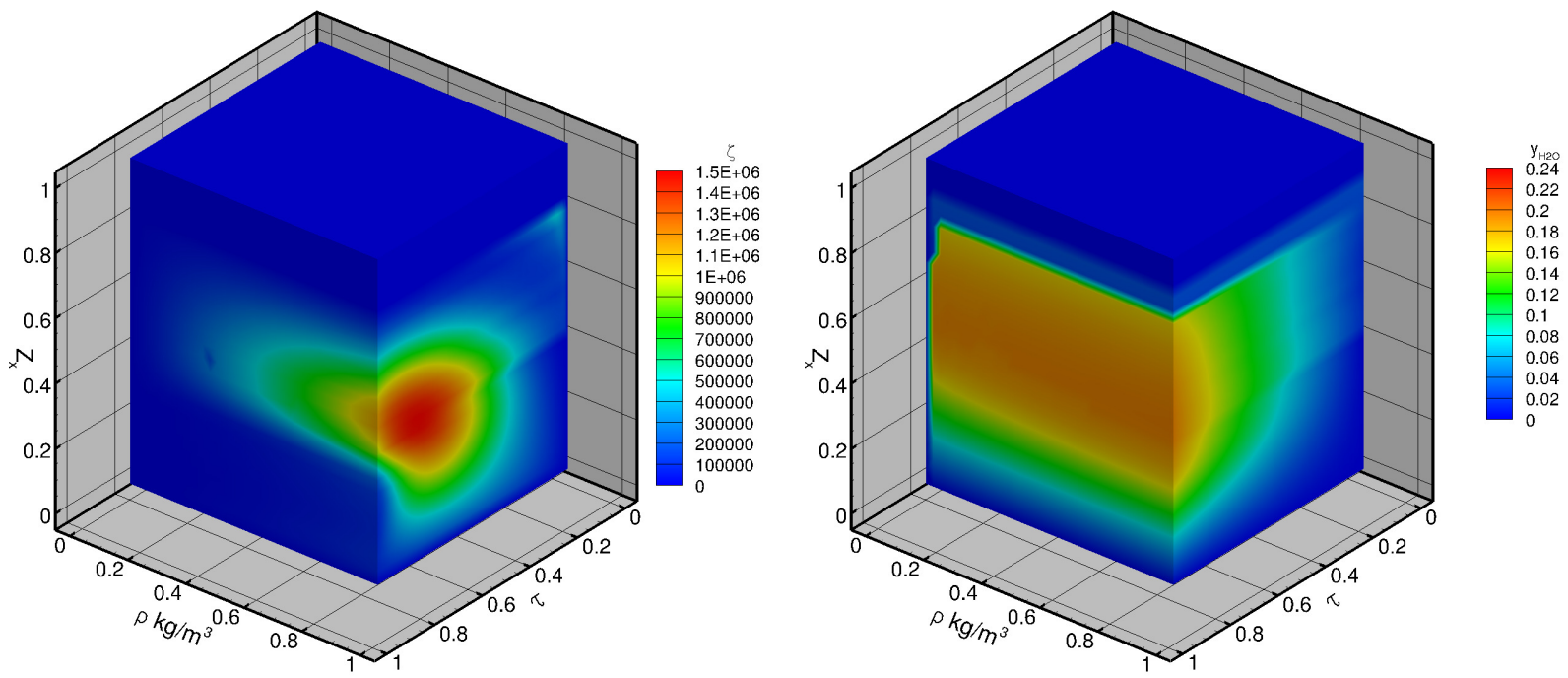

Figure 1. A portion of the EVM table for the Gamba experiments at a constant value of energy $(e=0.81 \mathrm{MJ} / \mathrm{kg})$; $\zeta$ (left) and mass fraction of $\mathrm{H}_{2} \mathrm{O}$ (right).

Such an approach would have significant advantages over a full solution of the detailed chemical kinetics model. Rather than solving conservation equations for each chemical species and evaluating a large chemical source term, a greatly reduced set of governing equations would be solved. The explicit tracking of the induction and ignition process permits auto-ignition dominated combustion flows to be accurately represented. 
Furthermore, for complex hydrocarbons, experimental databases may be used to parameterize the induction rate and to characterize the ignition delay time scale. This approach implicitly includes the formation of radical species that lead to ignition, without the cost of developing and validating detailed kinetics models and solving for trace radicals in a CFD simulation. Ultimately, such an approach would accurately represent the ignition delay time with little computational expense.

\section{Governing Equations}

In this section, we discuss the governing equations used for the induction-evolution variable approach. As discussed above, the induction-evolution variable approach uniquely determine the thermo-chemical state from the pressure, enthalpy, mixture fraction, $Z$, and induction-evolution variable, $\tau$. The conservation equations for this flow field are

$$
\begin{aligned}
\frac{\partial \rho}{\partial t}+\frac{\partial}{\partial x_{j}}\left(\rho u_{j}\right) & =0 \\
\frac{\partial \rho Z}{\partial t}+\frac{\partial}{\partial x_{j}}\left(\rho Z\left(u_{j}+v_{Z j}\right)\right) & =0 \\
\frac{\partial \rho \tau}{\partial t}+\frac{\partial}{\partial x_{j}}\left(\rho \tau\left(u_{j}+v_{\tau j}\right)\right) & =\rho \zeta
\end{aligned}
$$

along with the usual momentum and energy conservation equations. Here, $v_{Z j}$ and $v_{\tau j}$ are the mass diffusion velocities due to molecular and turbulent transport. The source term on the $\rho \tau$ equation represents the rate of production of the induction-evolution variable, and is the integrand in Eq. (1).

Here, $\rho Z$ is the density of the elements comprising the fuel and its reaction products; we can define a second partial density of the gas mixture as $\rho X=\rho-\rho Z$, which is the density of the gas mixture that is not composed of fuel and its reaction products. Let us define $\alpha_{s}$, which is the mass fraction of the fuel elements within a chemical species, $s$. Then

$$
\begin{aligned}
\rho Z & =\sum_{s} \rho_{s} \alpha_{s} \\
\rho X & =\sum_{s} \rho_{s}\left(1-\alpha_{s}\right)
\end{aligned}
$$

where the summation is over all species present in the gas mixture. The conservation equation for $\rho X$ is

$$
\frac{\partial \rho X}{\partial t}+\frac{\partial}{\partial x_{j}}\left(\rho X\left(u_{j}+v_{X_{j}}\right)\right)=0
$$

with $v_{X j}=-v_{Z j}$ for mass conservation. In the following, we use the $\rho X$ variable because it simplifies the derivation of the flux Jacobian and the formulation of the numerical flux function. Also, the resulting flux Jacobian has a form similar to that obtained for a mixture of reacting gases. ${ }^{10}$

Thus, the vector of conserved quantities and the corresponding convective flux vector for the threedimensional flow are

$$
\begin{aligned}
U & =(\rho X, \rho Z, \rho \tau, \rho u, \rho v, \rho w, E)^{T} \\
F & =\left(\rho X u^{\prime}, \rho Z u^{\prime}, \rho \tau u^{\prime}, \rho u u^{\prime}+p n_{x}, \rho v u^{\prime}+p n_{y}, \rho w u^{\prime}+p n_{z},(E+p) u^{\prime}\right)^{T}
\end{aligned}
$$

where $u^{\prime}=\vec{u} \cdot \hat{n}$ is the velocity normal to a cell face with unit normal vector $\hat{n}$. The thermo-chemical state is obtained from the tabulated evolution-variable manifold as discussed above and in detail by Cymbalist and Dimotakis.

\section{Numerical Method}

In this section, we discuss an approach to obtaining a self-consistent numerical flux function for the induction-evolution variable approach based on a set of tabulated thermodynamic data. First, we develop the convective flux Jacobian required for an upwind-biased flux, then discuss an approach for obtaining second-order accurate fluxes, and finally provide a brief description of a low-dissipation centered flux function suitable for highly compressible flows. 


\section{IV.A. Upwind Numerical Flux Formulation}

Let us first consider an upwind flux formulation such as modified Steger-Warming flux-vector splitting ${ }^{11}$ or Roe flux-difference splitting. ${ }^{12}$ For LES applications, we use the dissipative portion of the upwind flux to stabilize the low-dissipation centered flux. For the upwind flux, we must diagonalize the flux Jacobian, $\frac{\partial F}{\partial U}$. This is straight-forward except for the derivatives of the pressure with respect to the conserved variables. Following the approach in Ref. 10, it can be shown that

$$
\begin{aligned}
\frac{\partial F}{\partial U}=R^{-1} \Lambda R= & \left(\begin{array}{c}
\frac{\rho X}{\rho} \hat{\lambda} / a^{2} \\
\frac{\rho Z}{\rho} \hat{\lambda} / a^{2} \\
\frac{\rho \tau}{\rho} \hat{\lambda} / a^{2} \\
\left(u \hat{\lambda}+a n_{x} \tilde{\lambda}\right) / a^{2} \\
\left(v \hat{\lambda}+a n_{y} \tilde{\lambda}\right) / a^{2} \\
\left(w \hat{\lambda}+a n_{z} \tilde{\lambda}\right) / a^{2} \\
\left(h_{o} \hat{\lambda}+a u^{\prime} \tilde{\lambda}\right) / a^{2}
\end{array}\right) \\
+ & \left(\begin{array}{c}
p_{\rho X} \\
\frac{\rho X}{\rho} \tilde{\lambda} / a \\
\frac{\rho Z}{\rho} \tilde{\lambda} / a \\
\frac{\rho \tau}{\rho} \tilde{\lambda} / a \\
u \tilde{\lambda} / a+n_{x} \hat{\lambda} \\
v \tilde{\lambda} / a+n_{y} \hat{\lambda} \\
w \tilde{\lambda} / a+n_{z} \hat{\lambda} \\
h_{o} \tilde{\lambda} / a+u^{\prime} \hat{\lambda}
\end{array}\right)\left(\begin{array}{llllll}
-u^{\prime} & 0 & -u p_{E} & -v p_{E} & -w p_{E} & p_{E}
\end{array}\right)
\end{aligned}
$$

Where the diagonal matrix of eigenvalues is given as

$$
\Lambda=\operatorname{diag}\left(\lambda, \lambda, \lambda, \lambda^{+}, \lambda, \lambda, \lambda^{-}\right)
$$

where $\lambda=u^{\prime}, \lambda^{+}=u^{\prime}+a, \lambda^{-}=u^{\prime}-a, a$ is the speed of sound, and $\tilde{\lambda}$ and $\hat{\lambda}$ are defined as

$$
\tilde{\lambda}=\frac{1}{2}\left(\lambda^{+}-\lambda^{-}\right), \quad \hat{\lambda}=\frac{1}{2}\left(\lambda^{+}+\lambda^{-}-2 \lambda\right)
$$

and $h_{o}$ is the total enthalpy of the gas mixture.

The pressure derivatives

$$
p_{\rho X}=\frac{\partial p}{\partial \rho X}, \quad p_{\rho Z}=\frac{\partial p}{\partial \rho Z}, \quad p_{E}=\frac{\partial p}{\partial E}
$$

must be computed holding the conserved variables fixed. For a multi-species mixture of thermally-perfect gases, we have

$$
\left.\frac{\partial p}{\partial \rho_{s}}\right|_{\rho_{r \neq s}, \rho \vec{u}, E}=\frac{\hat{R}}{M_{s}} T+\frac{R}{c_{v}}\left(-e_{s}+\frac{1}{2} \vec{u} \cdot \vec{u}\right)
$$

where $R$ and $c_{v}$ are the mixture gas constant and specific heat

$$
R=\sum_{s} \frac{\rho_{s}}{\rho} \frac{\hat{R}}{M_{s}} T, \quad c_{v}=\sum_{s} \frac{\rho_{s}}{\rho} c_{v s}
$$

and $e_{s}$ is the energy of species $s$. Because $\rho X$ and $\rho Z$ are simply the weighted sum of the mixture species, we can construct their pressure derivatives with weighted sums of the local $X$ and $Z$ state.

$$
\begin{aligned}
\left.\rho X \frac{\partial p}{\partial \rho X}\right|_{\rho Z, \rho \tau, \rho \vec{u}, E} & =\sum_{s} \rho_{s}\left(1-\alpha_{s}\right) \frac{\partial p}{\partial \rho_{s}} \\
\left.\rho Z \frac{\partial p}{\partial \rho Z}\right|_{\rho X, \rho \tau, \rho \vec{u}, E} & =\sum_{s} \rho_{s} \alpha_{s} \frac{\partial p}{\partial \rho_{s}}
\end{aligned}
$$


In this case, $\rho \tau$ does not affect the pressure, and therefore its derivative is zero. The energy derivative is simply

$$
\left.\frac{\partial p}{\partial E}\right|_{\rho X, \rho Z, \rho \tau, \rho \vec{u}}=\frac{R}{c_{v}}
$$

Thus, we have obtained the convective flux Jacobian and we can formulate an upwind flux function. For example, the Roe flux is ${ }^{12}$

$$
F_{i+1 / 2}=\frac{1}{2}\left(F_{i}+F_{i+1}\right)-\frac{1}{2} \tilde{R}^{-1}|\tilde{\Lambda}| \tilde{R}\left(U_{i+1}-U_{i}\right)
$$

where the tilde-variables are Roe-averaged using the left and right data. For modified Steger-Warming flux vector splitting, ${ }^{11}$ the convective fluxes may be written as

$$
F_{i+1 / 2}=A_{i+1 / 2}^{+} U^{L}+A_{i+1 / 2}^{-} U^{R}
$$

Where $A^{ \pm}$are the Jacobians evaluated using the face data and the positive and negative eigenvalues, respectively.

The quantities $U^{L, R}$ are the conserved variables evaluated at the face using left and right biased data. For hypersonic and highly compressible flows, it is better to evaluate the primitive variables at the face and reconstruct the face values of the conserved variables. Thus, with MUSCL-type limiting, ${ }^{13}$ we have

$$
\begin{aligned}
& V_{i+1 / 2}^{L}=V_{i}+\frac{1}{2} \lim \left(V_{i+1}-V_{i}, V_{i}-V_{i-1}\right) \\
& V_{i+1 / 2}^{R}=V_{i+1}-\frac{1}{2} \lim \left(V_{i+2}-V_{i+1}, V_{i+1}-V_{i}\right)
\end{aligned}
$$

where $V=(\rho X, \rho Z, \rho \tau, u, v, w, p)^{T}$. It is trivial to obtain all of the conserved variables from the primitives, except for $E^{L, R}$. With a conventional multi-species formulation, $E$ may be obtained from the face density, pressure and chemical state. Here, however, the gas composition is not available. Instead, $E$ may be obtained using a Taylor series expansion of the form (for the left-biased data)

$$
E^{L}=E_{i}+\left.\frac{\partial E}{\partial \rho X}\right|_{i} \delta \rho X^{L}+\left.\frac{\partial E}{\partial \rho Z}\right|_{i} \delta \rho Z^{L}+\left.\frac{\partial E}{\partial u}\right|_{i} \delta u^{L}+\left.\frac{\partial E}{\partial v}\right|_{i} \delta v^{L}+\left.\frac{\partial E}{\partial w}\right|_{i} \delta w^{L}+\left.\frac{\partial E}{\partial p}\right|_{i} \delta p^{L}
$$

where the partial derivatives are taken holding the primitive variables fixed, and $\delta V^{L}=V_{i+1 / 2}^{L}-V_{i}$. A similar expression is used for the right-biased data. Again, most of these $E$ derivatives are straight-forward except for the partial density derivatives. For species $s$, we have

$$
\left.\frac{\partial E}{\partial \rho_{s}}\right|_{\rho_{r \neq s}, \vec{u}, p}=e_{s}-\frac{c_{v}}{R} \frac{\hat{R}}{M_{s}} T+\frac{1}{2} \vec{u} \cdot \vec{u}
$$

This approach accounts for the variation in the thermodynamic state between the cell-centered data and the cell face.

\section{IV.B. Kinetic Energy Consistent Flux}

The LES results presented below were computed with a second-order accurate centered flux function that has been shown to have low levels of dissipation. The flux is formulated so that it is discretely consistent with the compressible kinetic energy equation. ${ }^{14}$ The dissipative component of the modified Steger-Warming flux discussed above is added to provide dissipation near shock waves and other strong gradients. The Ducros sensor ${ }^{15}$ is used to activate the dissipative flux through a weight function, $\alpha$. The numerical flux may be written as

$$
F_{i+1 / 2}=F_{\mathrm{KEC}, i+1 / 2}-\alpha \frac{1}{2} R^{-1}|\Lambda| R\left(U_{i+1}-U_{i}\right)
$$

Where the centered flux is

$$
F_{\mathrm{KEC}, i+1 / 2}=\left(\begin{array}{c}
\bar{\rho} \bar{u}^{\prime} \bar{y} \\
\bar{\rho} \bar{u}_{s}^{\prime} u+\bar{p} n_{x} \\
\bar{\rho} \bar{u}^{\prime} v+\bar{p} n_{y} \\
\bar{\rho} \bar{u}^{\prime} w+\bar{p} n_{z} \\
\bar{\rho} \bar{u}^{\prime}(\bar{e}+\bar{k})+\bar{p} \bar{u}^{\prime}
\end{array}\right)
$$


Here the bars indicate and average between the left and right data, such as $\bar{\rho}=\frac{1}{2}\left(\rho^{L}+\rho^{R}\right)$; for the kinetic energy we use

$$
\bar{k}=\frac{1}{2}\left(u^{L} u^{R}+v^{L} v^{R}+w^{L} w^{R}\right)
$$

For second order, the nearest data are used, for example $\bar{\rho}=\frac{1}{2}\left(\rho_{i}+\rho_{i+1}\right)$; for a higher-order flux formulation, gradients may be used to obtain a more accurate representation of the flux.

\section{IV.C. Time Integration}

The results presented below use a grid that resolves the near-wall boundary layer, rather than using a wall model and coarse near-wall grid spacing. This approach imposes stringent time-step limitations; also, the source term for the evolution variable, $\tau$, may be large, imposing an additional restriction on explicit time integration methods. Therefore, implicit time integration methods are used. For Reynolds-averaged Navier-Stokes (RANS) simulations, the data-parallel line-relaxation method ${ }^{16}$ is used, and for large-eddy simulations (LES), a point-relaxation method ${ }^{17}$ is employed. Derivatives of the induction-evolution variable source term, $\zeta$, are tabulated and are used to form the source term Jacobian matrix in the implicit operator.

\section{EVM Implementation}

In the previous section, we discussed the development of a numerical flux function for a general mixture of gases, along with an induction-evolution variable, $\tau$. The state of this gas is represented by a table constructed at conditions relevant for a given range of flow conditions and fuel/oxidizer composition. As discussed above, any thermodynamic quantity can be tabulated and used to close the governing equations.

From the preceding discussion, in order to construct the self-consistent numerical flux, the EVM table must provide values for

$$
p, a, R, c_{v}, h, \frac{\partial p}{\partial \rho X}, \frac{\partial p}{\partial \rho Z}, \frac{\partial E}{\partial \rho X}, \frac{\partial E}{\partial \rho Z}
$$

as well as $\zeta$ and the mixture transport properties required for the diffusive flux calculation. The values of these quantities are then interpolated from the EVM table. An alternative approach would be to tabulate the composition (mass fraction) of the fuel-air mixture as a function of $\rho, e, Z, \tau$, and then construct the above thermodynamic variables using thermodynamic curve fits for the individual species. In the present work, we use the latter approach, and tabulate $\zeta$ and the 11 species mass fractions that represent the gas state for the Gamba et al. experiments and the Burke et al. kinetics model for hydrogen-air combustion.

The possible range of thermodynamic conditions in the experimental flow field is very large, particularly because the high-pressure hydrogen fuel overexpands to a Mach number of 5.7. This results in a range of pressure between $950 \mathrm{~Pa}$ and $2.47 \mathrm{MPa}$, and a range of temperatures between $48 \mathrm{~K}$ and $2850 \mathrm{~K}$. In order to cover this range of conditions, the EVM table was constructed for values of density between $0.001 \mathrm{~kg} / \mathrm{m}^{3}$ and $2.651 \mathrm{~kg} / \mathrm{m}^{3}$ and energies between $-4.01 \mathrm{MJ} / \mathrm{kg}$ and $9.61 \mathrm{MJ} / \mathrm{kg}$ (referenced to $T=298.15 \mathrm{~K}$ ). To better represent the variation of gas state with mixture fraction, the table was generated using the molar mixture fraction; the mass-based mixture fraction, $Z$, is then interpolated to its molar value. Non-uniform spacing in $\rho$ and $e$ was used to focus the table to best represent the bulk of the flow field in which reactions are occurring. Coarse spacing in $\rho$ and $e$ is used where little reaction occurs. The EVM table used for the following simulations discretizes $Z$ into 26 segments, $\tau$ into 100, $\rho$ into 36, and $e$ into 76 .

The EVM table is four-dimensional, and a general search in this domain would be expensive. However, it is stored as a 4-dimensional array, and the relationship between the values of the variables $(\rho, e, Z, \tau)$ and their indices are known. Thus, simple integer math produces the bounding indices of the location in the table, and then a tetra-linear interpolation is performed to obtain the values of $\zeta$ and the mass fractions. The table is stored as single precision (32-bit) real values to reduce its size.

\section{Test Case}

The EVM approach was used to simulate the hydrogen normal injection experiments of Gamba et al. at the high jet momentum flux condition; see Saghafian et al. ${ }^{4}$ for corresponding compressible flamelet progress variable simulations of this case. The computational domain was chosen to resolve the incoming boundary layer; the injection plenum was gridded, and embedded refinement regions were included near the jet, in the 
jet interaction region, and in the jet plume. The near-wall grid was clustered to resolve the boundary layer, with the near-wall spacing corresponding to $y^{+} \simeq 1$.

Figure 2 shows the computational domain, along with a temperature isosurface to visualize the jet plume. Figure 3 shows two images of the grid, with the embedded refinement regions. Note the use of 3-point and 5point singularities that allow the grid resolution to smoothly diminish away from the jet and plume. The grid was generated using the GoHypersonic Inc. LINK3D software. There are a total of 7.1 million hexahedral elements in the grid.

The flow conditions are taken from Gamba et al. as $T_{\infty}=1500 \mathrm{~K}, p_{\infty}=40 \mathrm{kPa}$, and $M_{\infty}=2.4$. This corresponds to a free-stream density of $0.0929 \mathrm{~kg} / \mathrm{m}^{3}$ and a Reynolds number of 6600 based on the $2 \mathrm{~mm}$ diameter jet and freestream conditions. The hydrogen plenum stagnation pressure and temperature were set to $2.46 \mathrm{MPa}$, and $300 \mathrm{~K}$. An isothermal surface temperature boundary condition at $300 \mathrm{~K}$ was assumed to represent the short-duration shock tube operation of the experiment.

Four simulations were run. Two used the full 11-species, 23-reaction kinetics model of Burke et al.; one was run in RANS mode using the Spalart-Allmaras turbulence model ${ }^{18}$ with the Catris-Aupoix ${ }^{19}$ compressibility correction. It used large time steps and a second-order accurate upwind flux. The second case was run as a wall-modeled LES (WM-LES) using the Improved Delayed Detached Eddy Simulation (IDDES) method of Shur et al. ${ }^{20}$ and the second-order accurate kinetic energy consistent low-dissipation fluxes. IDDES in combination with low-dissipation fluxes has been shown to compare well with normal injection into supersonic crossflow mixing experiments. ${ }^{21}$ Effectively, it uses the Spalart-Allmaras RANS model as a wall model, and a Smagorinsky-like subgrid-scale model outside the wall boundary layer. Two simulations were run using the EVM approach; one in RANS mode and one in WM-LES mode. All simulations were performed with first-order accurate implicit time integration; for the RANS cases large time steps were used to rapidly obtain a steady-state solution. For LES, much smaller time steps were used, corresponding to a CFL of about 10 ( $\Delta t \simeq 2 \mathrm{~ns}$ ) for the detailed kinetics model and a CFL of 2 for the EVM method. The EVM implementation is not as stable as the full kinetics code; the reason for this is under investigation.

These simulations are designed to test the EVM implementation. They are not meant to represent the best possible simulation of this experimental configuration. The grid is too small and the embedded grid refinement region does not enclose the outer edge of the jet plume. A simple model for the molecular transport properties is used. The incoming boundary layer is turbulent, instead of laminar as in the experiment. And the time integration is only first-order accurate. All of these limitations will be addressed in future work.

Figures 4-8 visualize the simulations, and compare the detailed kinetics model with the EVM approach. Figure 4 plots the induction-evolution variable, $\tau$, for the EVM simulations. The RANS result is plotted on the left, and the WM-LES on the right. The RANS simulation has much less unsteadiness due to the more dissipative fluxes and the action of the S-A turbulence model in the entire domain. The evolution variable is large in the shear layers at the edges of the jet, near the wall, and on the centerline under the jet plume.

Figures 5-8 plot the mixture fraction, $Z$, temperature, $\mathrm{OH}$ mass fraction, and $\mathrm{H}_{2} \mathrm{O}$ mass fraction for the four cases computed. The upper left plot in each figure is the detailed kinetics model run in RANS mode; the lower left is the detailed model run in WM-LES mode; the upper right is RANS with EVM; and finally the lower right is WM-LES with EVM. The mixture fraction plots show that the two RANS results are very similar, with slightly larger values of $Z$ in the EVM simulation plume near the exit of the domain. The EVM WM-LES shows a larger range of length scales in the plume; this may be caused by small differences in heat release, or to due to differences in the numerics. Here, the EVM simulations are limited to smaller time steps than the detailed kinetics simulations. Large time steps can effect the development of the shear layer, especially with the low-order time integration used here. More work is required to better characterize these differences.

The behavior of the mixture fraction are reflected in the other variables, $T, y_{\mathrm{OH}}$, and $y_{\mathrm{H}_{2} \mathrm{O}}$; in general there is strong similarity between the detailed kinetics and EVM. Again, differences are present due to the numerics, and more work is required to completely understand these effects. The largest difference is in the water mass fraction, with EVM producing little to no reaction in the jet plume, while the full kinetics model has significant $\mathrm{H}_{2} \mathrm{O}$ formation. Both of the WM-LES OH mass fraction plots show strong similarity with the experimental flow visualizations from Gamba et al. ${ }^{6,7}$

It is important to place these simulations in context - they are the first simulations to use a completely new combustion modeling approach. As such, the good agreement with the full kinetics model and the experimental data is promising. 


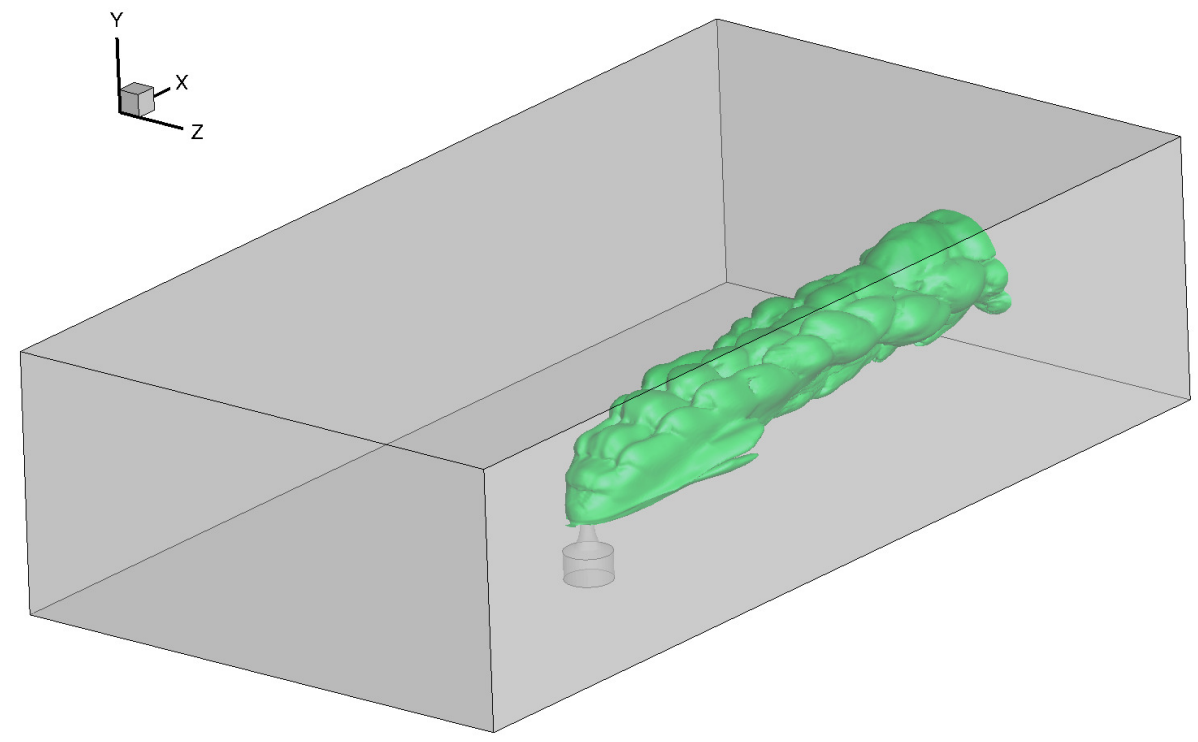

Figure 2. Flow field showing iso-surface of the temperature $(T=1400 \mathrm{~K})$ to visualize the flow field and solution domain.
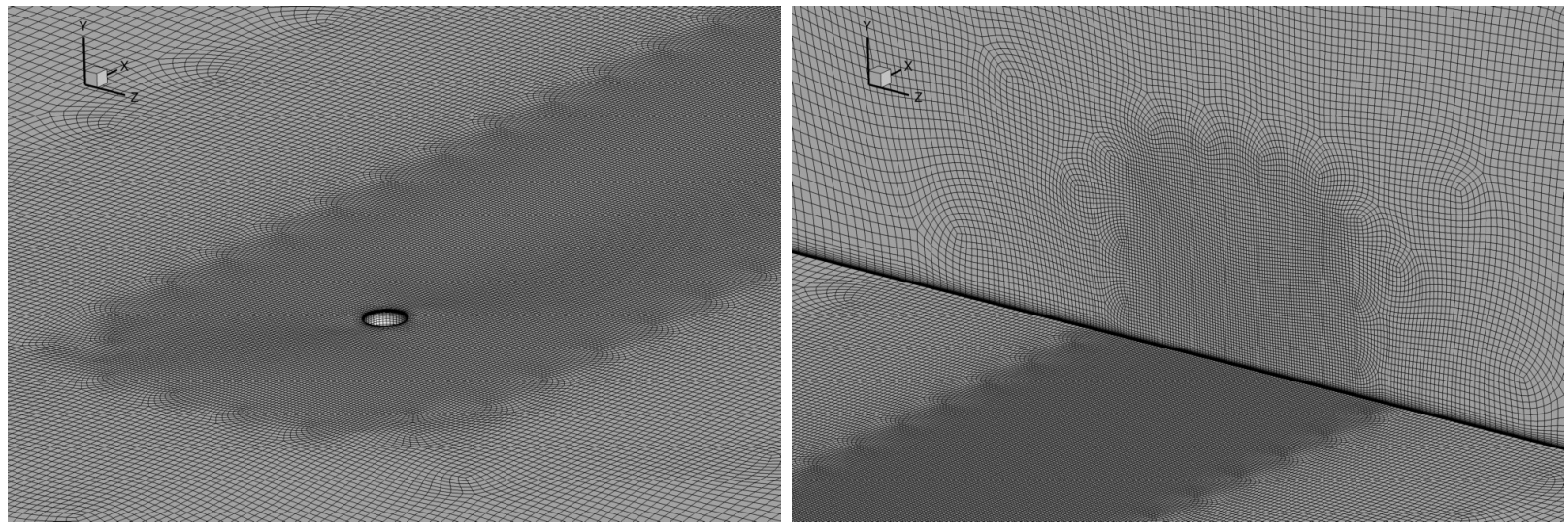

Figure 3. Computational grid used for the simulations; surface grid near injector (left) and surface and outflow grid (right). 


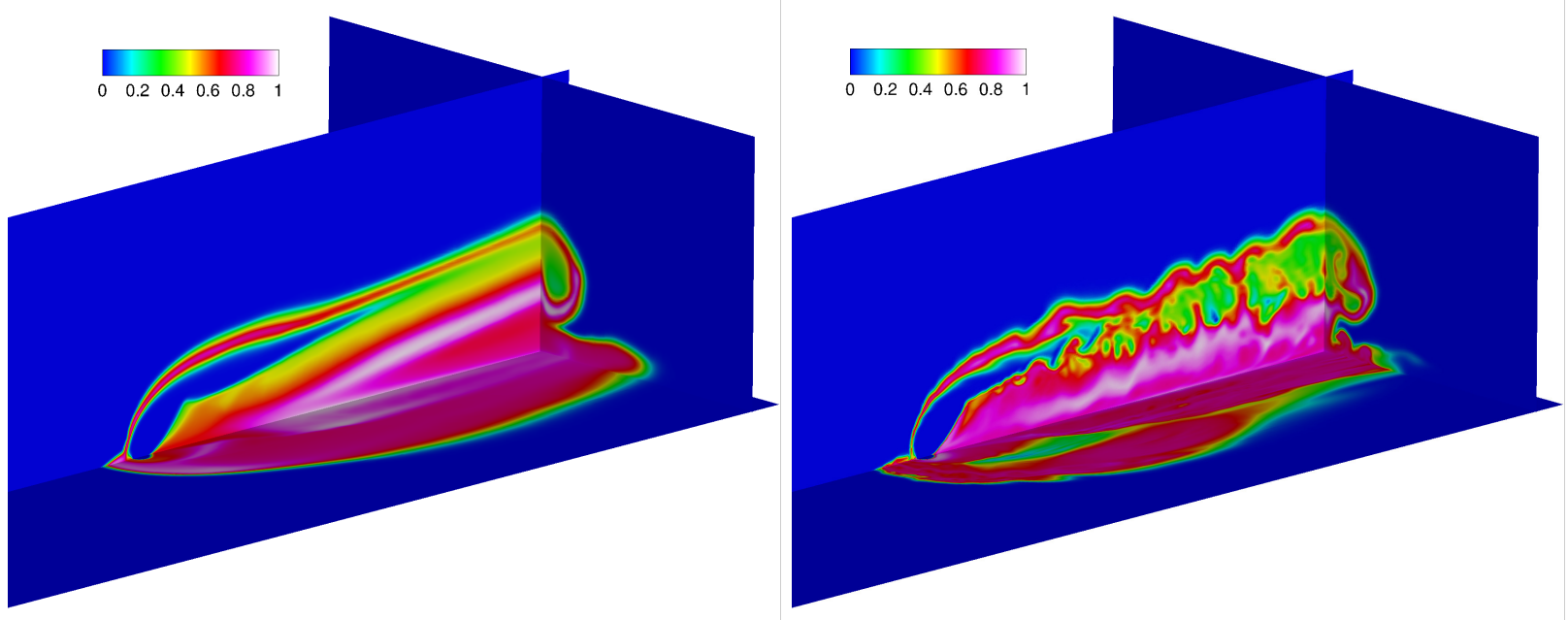

Figure 4. Variation of the induction-evolution variable, $\tau$, for RANS (left) and WM-LES (right).

\section{Conclusions and Future Work}

The evolution variable manifold approach of Cymbalist and Dimotakis ${ }^{1,2}$ has been implemented in a parallel implicit unstructured grid CFD code and compared with an experimental test case. A thermodynamicallyconsistent numerical flux function was formulated for dissipative upwind-biased fluxes and low-dissipation centered fluxes. The EVM table was used to provide the chemical composition and rate of change of the evolution variable as a function of the computed density, energy, mixture fraction, and evolution variable. The thermodynamic state is then computed directly from the interpolated composition from the EVM table.

Preliminary results for the hydrogen injection experiments of Gamba et al. ${ }^{6,7}$ were obtained with the EVM method, run in both RANS and LES mode. These simulations are not designed to represent the state of the art, rather they are meant to be used to test the implementation of the EVM method and assess its performance. Corresponding simulations using the full 11-species chemical kinetics model of Burke et al. ${ }^{8}$ were also performed.

The EVM simulations are in generally good agreement with the full kinetics model. The main difference observed is in the formation of the primary combustion product, with the EVM approach producing less product formation in the core of the jet plume. The EVM numerical implementation is less stable and cannot be run at extreme time steps as is the case for the simulations using the full kinetics model. The reason for this behavior is under investigation, but some numerical fluctuations in the EVM solutions may point to its cause.

The EVM approach shows promise for the simulation of high-speed flows that are dominated by ignition delay effects and auto-ignition. The preliminary simulations, while still a work in progress, illustrate that the approach may be implemented in a production CFD code and used to analyze realistic configurations. Work continues to compare the EVM model with more established turbulent combustion modeling approaches.

\section{Acknowledgments}

This work was sponsored by the Air Force Office of Scientific Research under grants FA9550-12-1-0064. The views and conclusions contained herein are those of the author and should not be interpreted as necessarily representing the official policies or endorsements, either expressed or implied, of the AFOSR or the U.S. Government. 

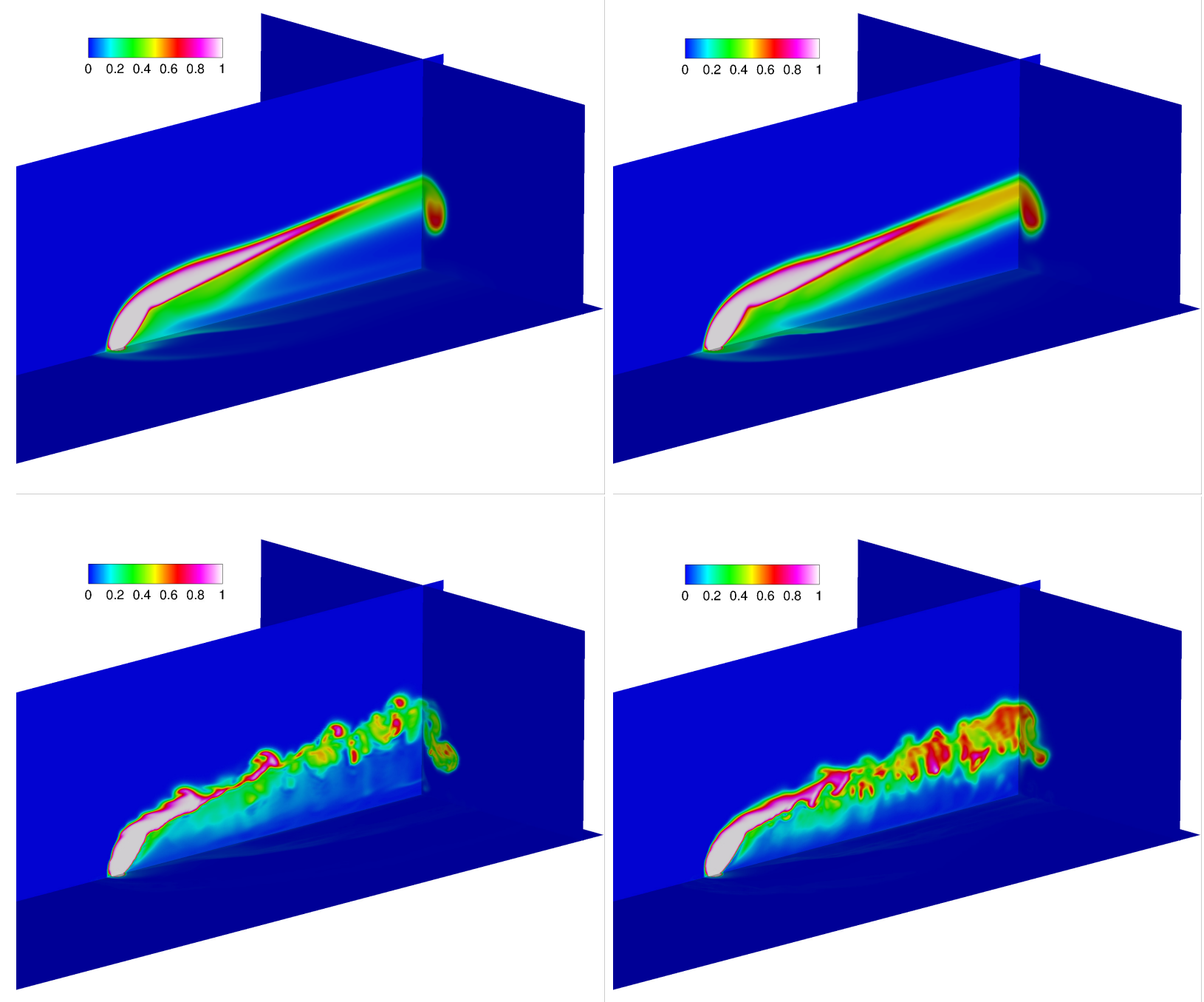

Figure 5. Mixture fraction, $Z$. Full kinetics model (left); EVM (right). RANS (top); WM-LES (bottom). 

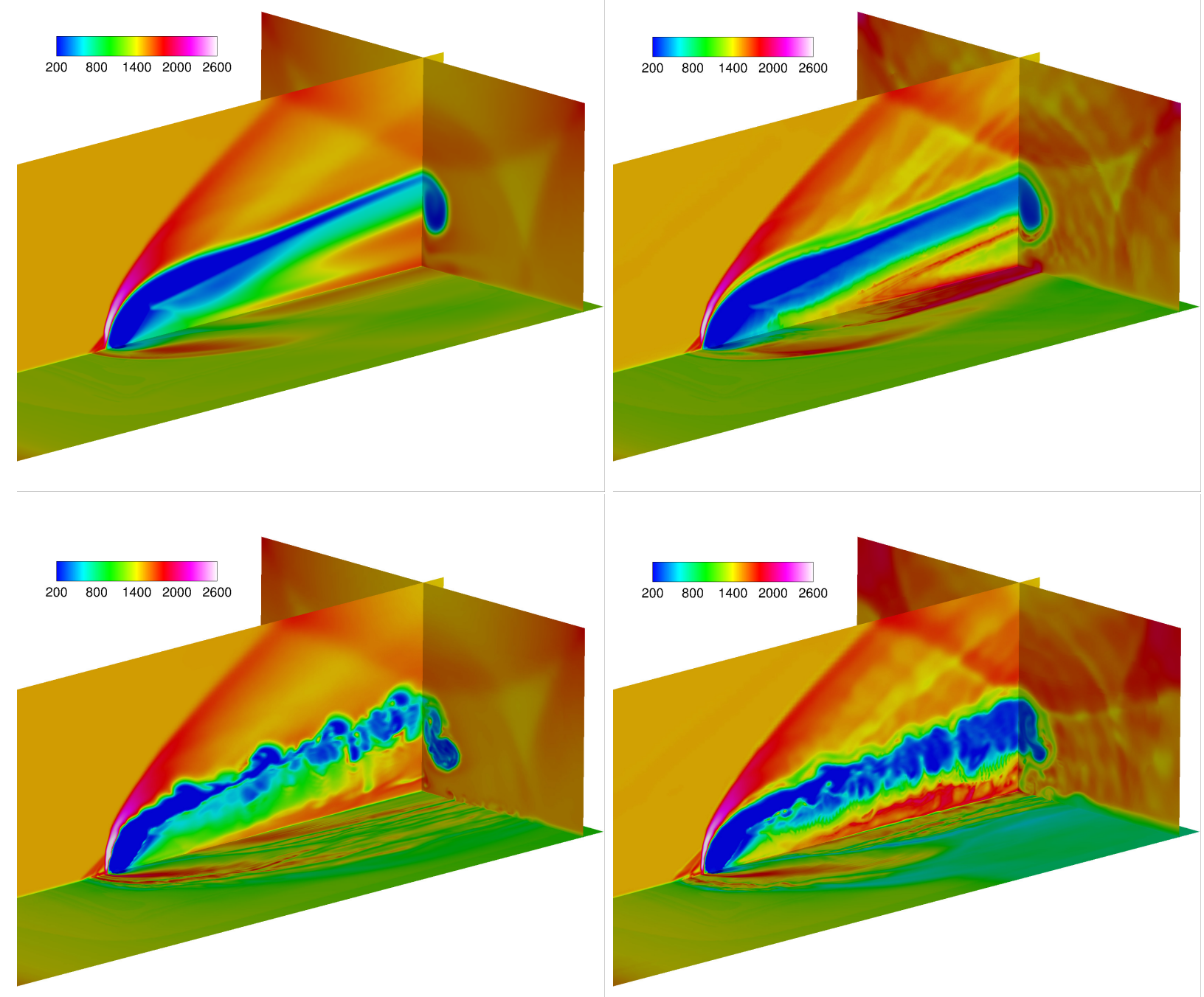

Figure 6. Temperature. Full kinetics model (left); EVM (right). RANS (top); WM-LES (bottom). 

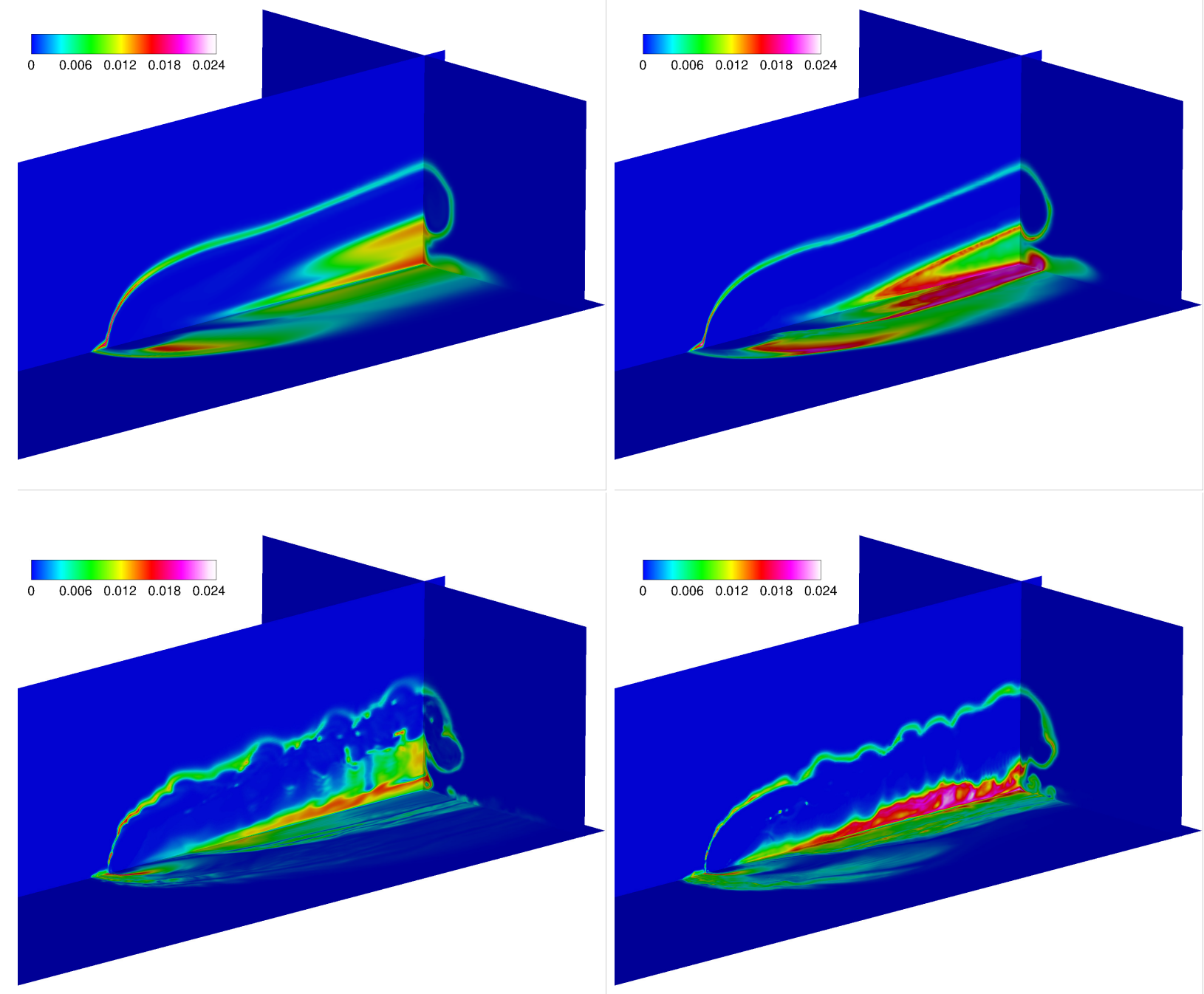

Figure 7. OH mass fraction. Full kinetics model (left); EVM (right). RANS (top); WM-LES (bottom). 

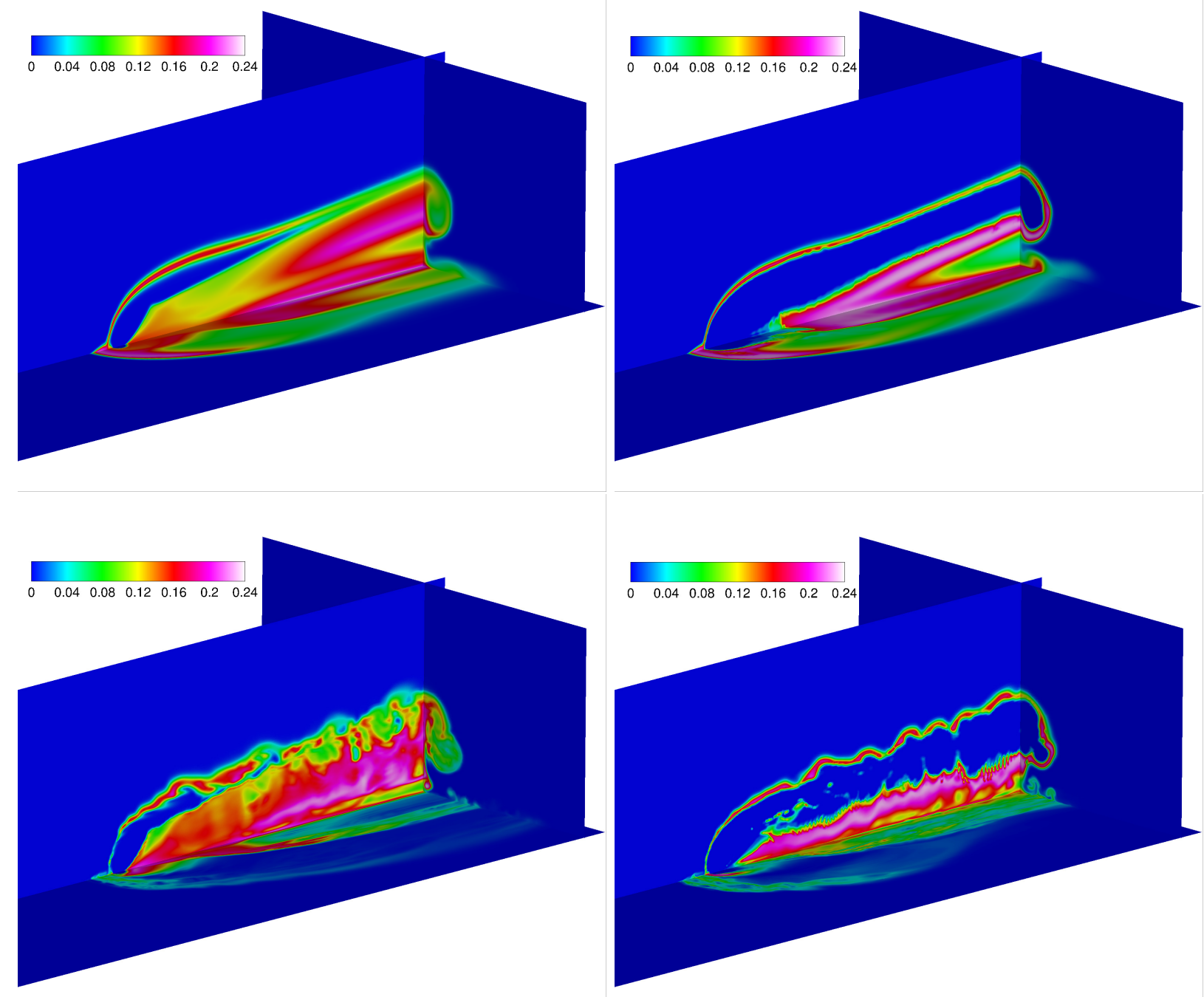

Figure 8. $\mathrm{H}_{2} \mathrm{O}$ mass fraction. Full kinetics model (left); EVM (right). RANS (top); WM-LES (bottom). 


\section{References}

${ }^{1}$ Cymbalist, N., and P.E. Dimotakis, "On Autoignition-Dominated Supersonic Combustion," AIAA Fluid Dynamics Conference, June 2015.

${ }^{2}$ Cymbalist, N., and P.E. Dimotakis, "Evolution-Variable Manifold model for Autoignition-Dominated Distributed Reactions." AIAA Journal, submitted 2015.

${ }^{3}$ Pierce, C.D., and P. Moin, "Progress-Variable Approach for Large-Eddy Simulation of Non-Premixed Turbulent Combustion," Journal of Fluid Mechanics, Vol. 204, pp. 73-97, 2004.

${ }^{4}$ Saghafian, A., V.E. Terrapon, and H. Pitsch, "An Efficient Flamelet-Based Combustion Model for Compressible Flows," Combustion and Flame, Vol. 162, Issue 3, pp. 652-667, March 2015.

${ }^{5}$ Candler, G.V., H.B. Johnson, I. Nompelis, P.K. Subbareddy, V. Gidzak, and M.D. Barnhardt, "Development of the US3D Code for Advanced Compressible and Reacting Flow Simulations," AIAA SciTech, Jan. 2015.

${ }^{6}$ Gamba, M., M.G. Mungal, and R.K. Hanson, "Ignition and Near-Wall Burning in Transverse Hydrogen Jets in Supersonic Crossflow," AIAA 2011-319, Jan. 2011.

${ }^{7}$ Gamba, M., V.E. Terrapon, A. Saghafian, M.G. Mungal, and H. Pitsch, "Assessment of the Combustion Characteristics of Hydrogen Transverse Jets in Supersonic Crossflow," Center for Turbulence Research Annual Research Briefs 2011, pp. 259-272.

${ }^{8}$ Burke, M.P., M. Chaos, Y. Ju, F.L. Dryer, and S.J. Klippenstein, "Comprehensive $\mathrm{H}_{2} / \mathrm{O}_{2}$ Kinetic Model for High-Pressure Combustion," International Journal of Chemical Kinetics, Vol. 44, pp. 444-474, 2012.

${ }^{9}$ Williams, F.A., "Atomization Processes and Ignition Criteria for Supersonic Combustion with Liquid Fuel Injection," Astronautica Acta, Vol. 15, pp. 547, 1970.

${ }^{10}$ Candler, G.V., P.K. Subbareddy, and J.M. Brock, "Advances in Computational Fluid Dynamics Methods for Hypersonic Flows," J. Spacecraft and Rockets, Vol. 52, No. 1, pp. 17-28, Jan.-Feb. 2015.

${ }^{11}$ MacCormack, R.W., Numerical Computation of Compressible and Viscous Flow, AIAA Education Series, 2014.

${ }^{12}$ Roe, P., "Characteristic-Based Schemes for the Euler Equations," Annual Review of Fluid Mechanics, Vol. 18, pp. 337$365,1986$.

${ }^{13}$ van Leer, B., "Towards the Ultimate Conservative Difference Scheme, V. A Second Order Sequel to Godunov's Method," J. Computational Physics, Vol. 32, pp. 101-136, 1979.

${ }^{14}$ Subbareddy, P., and G.V. Candler, "A Fully-Discrete, Kinetic Energy Consistent Finite Volume Scheme for Compressible Flows," J. Computational Physics, Vol. 228, pp. 1347-1364, Mar. 2009.

${ }^{15}$ Ducros, F., V. Ferrand, F. Nicoud, C. Weber, D. Darracq, C. Gacherieu, T. Poinsot, "Large-Eddy Simulation of Shock/Turbulence Interaction," J. Computational Physics, Vol. 152, No. 2 pp. 517-549, 1999.

${ }^{16}$ Wright, M.J., D. Bose, and G.V. Candler, "A Data-Parallel Line Relaxation Method for the Navier-Stokes Equations," AIAA Journal, Vol. 36, No. 9, pp. 1603-1609, Sept. 1998.

${ }^{17}$ Wright, M.J., G.V. Candler, and M. Prampolini, "Data Parallel Lower-Upper Relaxation Method for the Navier-Stokes Equations," AIAA Journal, Vol. 34, No. 7, pp. 1371-1377, July 1996.

${ }^{18}$ Spalart, P.R., and Allmaras, S.R., "A One-Equation Turbulence Model for Aerodynamic Flows," AIAA Paper 92-0439, Jan. 1992.

${ }^{19}$ Catris, S., and B. Aupoix, "Density Corrections for Turbulence Models," Aerospace Science and Technology, Vol. 4, 2000, pp. 1-11.

${ }^{20}$ Shur, M. L., P.R. Spalart, M.K. Strelets, and A.K. Travin, "A Hybrid RANS-LES Approach with Delayed-DES and Wall-Modelled LES Capabilities," Int. J. Heat and Fluid Flow, Vol 29, No. 6, pp. 16381649, 2008.

${ }^{21}$ Peterson, D.M., G.V. Candler, "Hybrid Reynolds-Averaged and Large-Eddy Simulation of Normal Injection into a Supersonic Crossflow," J. Propulsion and Power, Vol. 26, No. 3, pp. 533-544, 2010. 